\title{
Constrangimentos espácio-temporais sobre a tomada de decisão do tipo de remate na grande área do futebol
}

\author{
Life Spatiotemporal constraints on the decision-making of type of kick in the \\ soccer goal area
}

\author{
Fabian Alberto Romero Clavijo ${ }^{1 *}$, Renata Alvares Denardi ${ }^{1}$, Bruno Travassos ${ }^{2,3}$, Umberto \\ Cesar Corrêa ${ }^{1}$
}

ARTIGO ORIGINAL | ORIGINAL ARTICLE

\begin{abstract}
O presente estudo investigou a tomada de decisão da trajetória da bola e da superfície de contato do pé com a bola no remate do futebol. Foram filmados 12 jogos de futebol de um campeonato amador na Colômbia. Participaram no estudo 150 jogadores do sexo masculino, com idade entre 17 e 38 anos, e experiência de prática neste esporte de, no mínimo, 7 anos. As imagens capturadas foram analisadas através do software TACTO, e as variáveis espácio-temporais consideradas para análise foram as seguintes: I) ângulo de remate; II) distância entre o atacante e o defensor (interpessoal), III) distância entre o defensor e a baliza (extrapessoal); IV) velocidade e variabilidade de cada uma dessas medidas. Essas variáveis foram analisadas em relação a situações de remates com trajetórias retilíneas e parabólicas da bola, assim como, com a parte interna e peito do pé. Os resultados revelaram que a escolha da trajetória da bola foi influenciada pelas variáveis ângulo de remate e distância interpessoal. Além disso, a escolha da superfície de contato foi influenciada pela variabilidade da distância extrapessoal. Concluiu-se que as escolhas da trajetória da bola e da superfície de contato do remate são influenciadas pela interação espacial entre os jogadores.

Palavras-chave: tomada de decisão, futebol, dinâmica ecológica, remate.
\end{abstract}

\begin{abstract}
This study investigated the soccer players' decision-making of kicking using different ball trajectories and different foot-ball contact surfaces. Soccer kick events from 12 games of an amateur Colombian championship were selected and analysed using TACTO software. Participants included 150 male players aged between 17-38 years possessing at least 7 years of experience. The spatiotemporal variables considered were: the angle of the kick, the distance between the attacker and the defender (interpersonal) and between the defender and the goal (extra personal), and also the velocity and variability of each variable. The analysis included rectilinear and parabolic ball trajectories shots, and inside of the foot and instep kicks. The results revealed that the choice of kicking using a rectilinear or parabolic trajectory was influenced by the angle of the kick and the interpersonal distance. Inside of the foot or instep kicks were chosen depending on the variability of the extra personal distance. The findings highlight that the choice of kicking using different ball trajectories and different parts of the foot is influenced by spatial interactions between players.

Keywords: decision-making, soccer, ecological dynamic, kick.
\end{abstract}

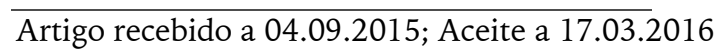

${ }^{1}$ Laboratório de Comportamento Motor, Escola de Educação Física e Esporte, Universidade de São Paulo, USP, São Paulo, Brasil

2 Departamento de Ciências do Desporto, Universidade de Beira Interior, Covilhã, Portugal

${ }^{3}$ CIDESD - Centro de Investigação em Desporto, Saúde e Desenvolvimento Humano, CreativeLab, Vila Real, Portugal

* Autor correspondente: Universidade de São Paulo, Escola de Educação Física e Esporte, Av. Mello Moraes, 65, Cidade Universitária, São Paulo, SP, Brazil CEP 05508-030. E-mail: fromero@usp.br 


\section{INTRODUÇÃO}

Nos últimos anos, um número considerável de pesquisas tem investigado os processos de tomada de decisão em modalidades esportivas coletivas de modo a obter uma melhor compreensão sobre os constrangimentos informacionais nas decisões dos jogadores (Corrêa, Davids, Silva, Denardi, \& Tani, 2014; Corrêa, Vilar, Davids, \& Renshaw, 2014; Correia et al., 2012; Vilar, Araújo, Davids, Correia, \& Esteves, 2013). De uma forma geral, em modalidades coletivas, a tomada de decisão pode ser definida como a escolha de uma determinada ação de entre várias possibilidades (Bar-Eli \& Raab, 2006). A tomada de decisão está relacionada à adaptação das ações dos indivíduos às constantes mudanças que ocorrem no jogo com alto grau de imprevisibilidade (Davids, 2009).

De entre as distintas perspectivas teóricas que permitem explicar o comportamento dos jogadores em contextos desportivos, recentemente vários estudos têm sido realizados em uma escala ecológica de análise, ou seja, considerando as decisões nos contextos em que elas ocorrem (Vilar, Araújo, Davids, \& Button, 2012). Esta perspectiva de dinâmica ecológica considera que as decisões são tomadas com base na percepção daquilo que o ambiente de jogo possibilita que se faça, centrando a sua atenção na identificação dos constrangimentos informacionais (relações espácio-temporais) que guiam a percepção e ação dos jogadores para a identificação de possibilidades de ação (Araújo, Davids, \& Hristovski, 2006)

Nesse contexto, uma gama de investigações tem sido realizada considerado variáveis físicas espácio-temporais como medidas da interação, por exemplo, entre os jogadores é chamada de interação interpessoal $\mathrm{e}$, entre jogadores $\mathrm{e}$ aspectos do ambiente de jogo (e.g., a baliza, as linhas do campo) é conhecida com interação extrapessoal, essas variáveis permitem especificar e justificar as decisões dos jogadores e equipas (Millar, Oldham, \& Renshaw, 2013). Como exemplo dessas variáveis podemos destacar a análise da variação de ângulos entre jogadores ou entre jogadores e a baliza (Corrêa, Vilar, Davids, \& Renshaw, 2012; Corrêa et al.,
2014; Travassos, Araújo, Duarte, \& McGarry, 2012), distâncias entre jogadores (Duarte, Freire, Gazimba, \& Araújo, 2010; Vilar et al., 2013) ou velocidades relativas entre jogadores (Duarte et al., 2010; Vilar, Araújo, Davids, \& Button, 2012). Assume-se, também, que valores específicos dessas variáveis atuam como parâmetros de controle sobre a tomada de decisão.

Tomando por base a perspectiva da dinâmica ecológica, boa parte dos estudos tem sido focada principalmente em uma classe de esportes caracterizada pela interação e distribuição dos participantes dentro de um espaço comum, os esportes de invasão. Por exemplo, verificam-se estudos realizados com basquetebol (Esteves, de Oliveira, \& Araújo, 2011), rúgbi (Correia et al., 2012; Passos, Cordovil, Fernandes, \& Barreiros, 2012) e futsal (Corrêa et al., 2012, 2014; Travassos et al., 2012; Vilar et al., 2013). Especificamente, os achados dos estudos supracitados evidenciam que as relações espaçotemporais entre jogadores e entre esses e aspectos do campo de jogo atuam como constrangimentos sobre a tomada de decisão de ações tais como, as velocidades e direções de execução do try, tackle, passe e remate. Um fator que chama a atenção na maioria dos citados estudos diz respeito à análise da tomada de decisão em locais específicos e reduzidos do campo de jogo, principalmente em lugares mais próximos onde acontecem as finalizações, e por um número reduzido de jogadores.

Apesar do número crescente de estudos sobre tomada de decisão em esportes coletivos e mais especificamente no futebol, a compreensão sobre grande parte dos contextos de jogo que caracterizam esta modalidade ainda se encontra por explorar. Por exemplo, Duarte et al. (2010) analisaram a tomada de decisão em situações 1 vs 1 , verificando que a distância interpessoal e a velocidade relativa entre jogadores são variáveis fundamentais para a compreensão da tomada de decisão do drible. De modo semelhante, Headrick et al. (2011) avaliaram qual a influência da distância à baliza na relação interpessoal gerada entre atacante e defensor para o drible no futebol. Esses estudos evidenciam a influência da relação entre velocidade relativa e a distância interpessoal, assim como da distância entre os 
jogadores e o gol no comportamento dos jogadores em situações de drible. A partir das evidências apresentadas, pode-se observar que não existem na literatura estudos analisando as variáveis espácio-temporais como informações que constrangem a ação do remate no futebol.

Em líneas gerais, pode-se visualizar uma limitação dos estudos no sentido de eles terem sido realizados em outras modalidades, outras ações de jogo e, por meio de situações adaptadas (1 vs 1). Também, pese a importância dos resultados desses estudos para o avanço do conhecimento sobre tomada de decisão no futebol, a quantidade de investigações presentes na literatura sobre esse tema é ainda insuficiente para generalizações. Além disso, tomando por base o pressuposto que o êxito no jogo de futebol é finalizar efetivamente, ou seja, marcar golo, e que devido à proximidade da grande área com a baliza, um elevado número de golos é realizado por meio de remates dentro deste setor específico do campo. Dessa maneira, reitera-se a importância de analisar a tomada de decisão do remate dentro da grande área. Face ao referido, este estudo tem como objetivo investigar relações espácio-temporais inter e extrapessoais como constrangimentos da tomada de decisão do remate no futebol. Especificamente, investigouse a influência do ângulo de remate, da distância interpessoal e da distância extrapessoal sobre tipo de remate utilizado dentro da grande área no futebol. O tipo de remate foi analisado em termos da trajetória da bola e da superfície de contato do pé com a bola. Tomando por base os resultados dos estudos realizados por Corrêa et al. (2012, 2014); Duarte et al. (2010); e Travassos et al. (2013), os quais encontraram que os valores dos ângulos e as distâncias influenciam na tomada de decisão dos jogadores, nossas hipóteses foram que os jogadores escolheriam (I) o remate retilíneo, quando os valores da lacuna angular na baliza fossem maiores; (II) o remate parabólico, quando não houvesse uma lacuna angular na baliza, ou ela fosse reduzida; (III) o remate com a parte interna ("chapa") do pé, quando as distâncias inter e extrapessoais fossem menores; e (IV) o remate com a parte superior do pé ("peito do pé"), nas situações em que as distâncias inter e extrapessoais fossem maiores.

\section{Participantes}

\section{MÉTODO}

O estudo foi realizado com 150 jogadores de futebol do sexo masculino, com idades compreendidas entre os 17 e os 38 anos, e experiência de prática neste esporte de, no mínimo, 7 anos. Todos os jogadores participaram do campeonato "Hexagonal del Olaya - 55 edição”, realizado na cidade de Bogotá- Colômbia em 2015. A coleta dos dados foi autorizada pela comissão organizadora do evento. $\mathrm{O}$ presente estudo foi aprovado pelo Comitê de Ética em Pesquisa da Escola de Educação Física e Esporte da Universidade de São Paulo.

\section{Coleta de dados}

A captura dos vídeos dos jogos foi realizada em 12 jogos, com duração de 90 minutos cada, por meio de duas câmeras digitais fixas (CASIO HS EX-FH100 - 10.1 megapixels) com uma frequência de $29 \mathrm{~Hz}$, localizadas nas arquibancadas lateralmente ao campo de futebol $\mathrm{e}$ às grandes áreas. Particularmente, foram capturados os deslocamentos de todos os jogadores e suas respectivas ações dentro das duas grandes áreas em jogadas que terminaram com remate. Das sequências de jogo filmadas, foram selecionadas as 70 jogadas em que o remate foi executado dentro da grande área. Os remates foram considerados em termos de trajetórias: I) retilínea $(\mathrm{n}=33)$, remates em que a bola manteve o contato com o gramado do campo ao longo da trajetória; e II) parabólica (n =37), remates em que a bola apresentou uma trajetória aérea depois do contato com o pé do jogador; e das superfícies de contato: I) parte interna do pé ("chapa") ( $=18)$; e II) parte superior do pé ("peito do pé") ( $=52)$.

\section{Análise dos dados}

Inicialmente, as imagens coletadas foram convertidas para uma frequência de $25 \mathrm{~Hz}$, sendo posteriormente digitalizadas no software TACTO (Fernandes, Folgado, Duarte, \& Malta, 2010). Para os procedimentos de digitalização, foi localizado o ponto entre os dois apoios (pés) de cada jogador dentro da grande área, considerando este lugar como a projeção do centro de gravidade sobre o chão (Duarte et al., 2010). A digitalização 
consistiu em seguir com o mouse a projeção do centro de gravidade de cada respetivo jogador sobre o chão, na imagem do vídeo em câmera lenta (frequência $=2 \mathrm{HZ}$ ). O software utilizado permitiu obter as trajetórias / deslocamentos dos jogadores em 2D, através das coordenadas $x$ e $y$ das posições de cada jogador dentro da grande área. Posteriormente, foi aplicado o método de reconstrução bidimensional por meio de transformação linear direta (2D-DLT), que possibilita converter as coordenadas virtuais (pixels) em coordenadas reais (metros). Para acessar ao nível de confiabilidade, realizou-se um teste de correlação dos rastreamentos realizados duas vezes pelo mesmo avaliador. O resultado $(\mathrm{r}$ $=0.98)$ indicou alto coeficiente de correlação intra-avaliador.

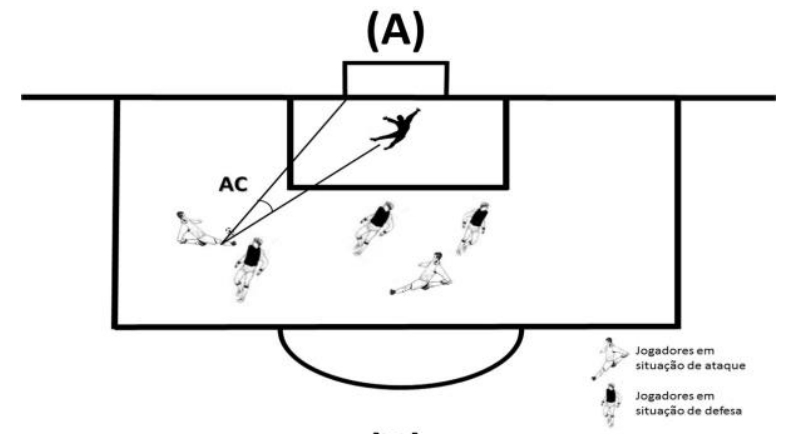

(B)

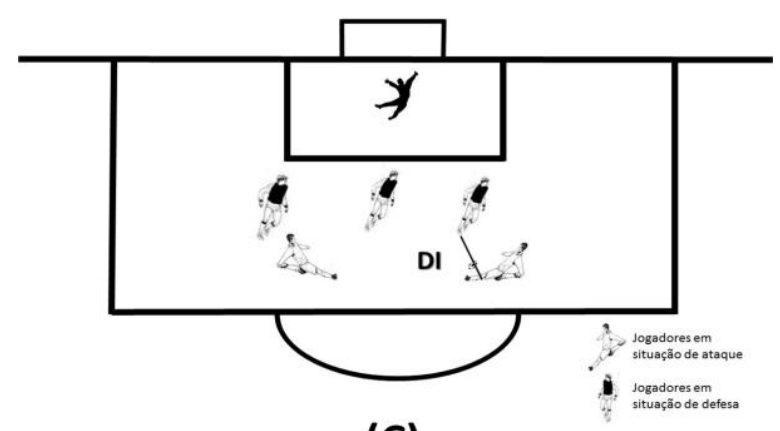

(c)

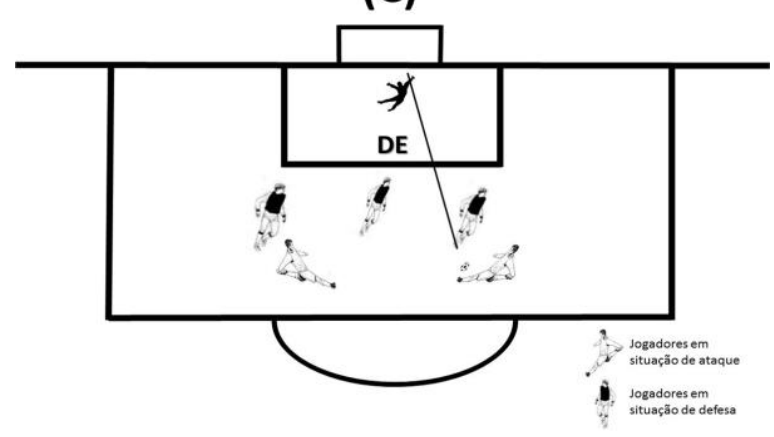

Figura 1. Ilustração do ângulo de remate (A), da distância interpessoal (B), e da distância extrapessoal (C)
Para examinar a influência das relações espácio-temporais na escolha do tipo de remate em relação à trajetória da bola e à parte do pé em contato com a bola, foram analisadas três variáveis:

1- Ângulo de remate (AR) (figura 1a): Vetores ligando o jogador com posse de bola (vértice) com dois componentes do jogo que geram a maior lacuna angular na baliza. Esses componentes de ligação com o portador da bola podem ser dois jogadores rivais, um jogador e uma trave ou as duas traves da baliza.

2- Distância interpessoal (DI) (figura 1b): Entre o atacante com posse de bola e o seu adversário mais próximo situado entre ele e a baliza.

3- Distância extrapessoal (DE) (figura 1c): Entre o adversário citado no item anterior e a linha da baliza.

As variáveis espácio-temporais foram analisadas a partir do momento em que o jogador fica em posse da bola (momento inicial) até o momento em que ele realiza o remate (momento final). As três supracitadas variáveis foram também analisadas em relação a duas taxas de mudança: velocidade e variabilidade entre o momento inicial e o momento final. No que se refere ao AR, primeiramente cada vetor foi obtido através da fórmula:

$$
a=\sqrt[2]{(\mathrm{P} 2 x-\mathrm{P} 1 x)^{2}+(\mathrm{P} 2 y-\mathrm{P} 1 y)^{2}},
$$

em que $a$ refere-se à distância entre o ponto 1 (P1) e o ponto 2 (P2).

Em seguida, o AR foi calculado por meio de:

$$
\operatorname{Cos} \theta=a^{2}-\left(b^{2}+c^{2}\right) /-2 . \text {. b.c. }
$$

A DI foi obtida através da formula:

$$
D I=\sqrt[2]{(\mathrm{P} 2 x-\mathrm{P} 1 x)^{2}+(\mathrm{P} 2 y-\mathrm{P} 1 y)^{2}},
$$

no qual P1 se refere à posição do atacante e P2 à do defensor. No mesmo sentido, a DE foi obtida através da fórmula:

$$
D E=\sqrt[2]{(\mathrm{P} 2 x-\mathrm{P} 1 x)^{2}+(\mathrm{P} 2 y-\mathrm{P} 1 y)^{2}},
$$


em que P1 se refere à posição do defensor e P2 à linha da baliza.

As variáveis anteriores foram também analisadas em relação à velocidade e variabilidade existente entre os dois momentos considerados. A velocidade do ângulo de remate e das distâncias foi calculada através da fórmula $v($ a) $=(F-I) /$ $t$, correspondendo $F$ ao valor de ângulo / distância no momento final (quando o portador de bola realiza o remate) e I ao valor de ângulo / distância no momento inicial (quando o jogador fica em posse da bola). Finalmente, a variabilidade em ambas as variáveis foi obtida por meio do cálculo do coeficiente de variação entre os 2 momentos previamente referidos $(C V(d)=$ $\sigma / \mu$, em que $C V$ é coeficiente de variação, $\sigma$ refere-se ao desvio padrão, e $\mu$ é a média).

\section{Tratamento estatístico}

Os valores iniciais e finais dos AR, das DI e das DE, bem como a velocidade e variabilidade de cada uma das variáveis foram comparados por meio de ANOVAs one-way em relação às trajetórias do remate (retilínea e parabólica), e à superfície de contato do pé utilizada em cada remate (interna ou peito do pé). No tratamento estatístico considerou-se o nível de significância de $p \leq 0.05$, e foi utilizado o software IBM SPSS statistics 20 .

\section{RESULTADOS}

\section{Trajetória da bola}

Os resultados mostraram diferenças significativas entre os remates com trajetórias retilínea e parabólica relativas ao ângulo inicial de remate $\left(F_{(1,68)}=6.95, p=0.01, \eta^{2}=0.09\right)$ e à DI inicial $\left(F_{(1,64)}=8.22, p=0.00, \eta^{2}=0.11\right)$. Conforme ilustra a Figura 2, os remates com trajetória retilínea foram executados quando os ângulos iniciais eram superiores e as distâncias interpessoais inferiores, ambos em comparação com os remates com trajetória parabólica.

Nenhuma outra diferença estatisticamente significativa foi encontrada nas demais variáveis.

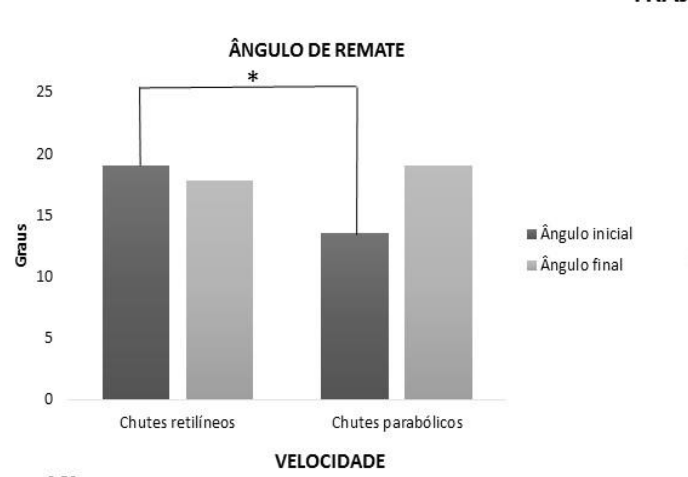

RAJETÓRIA DA BOLA
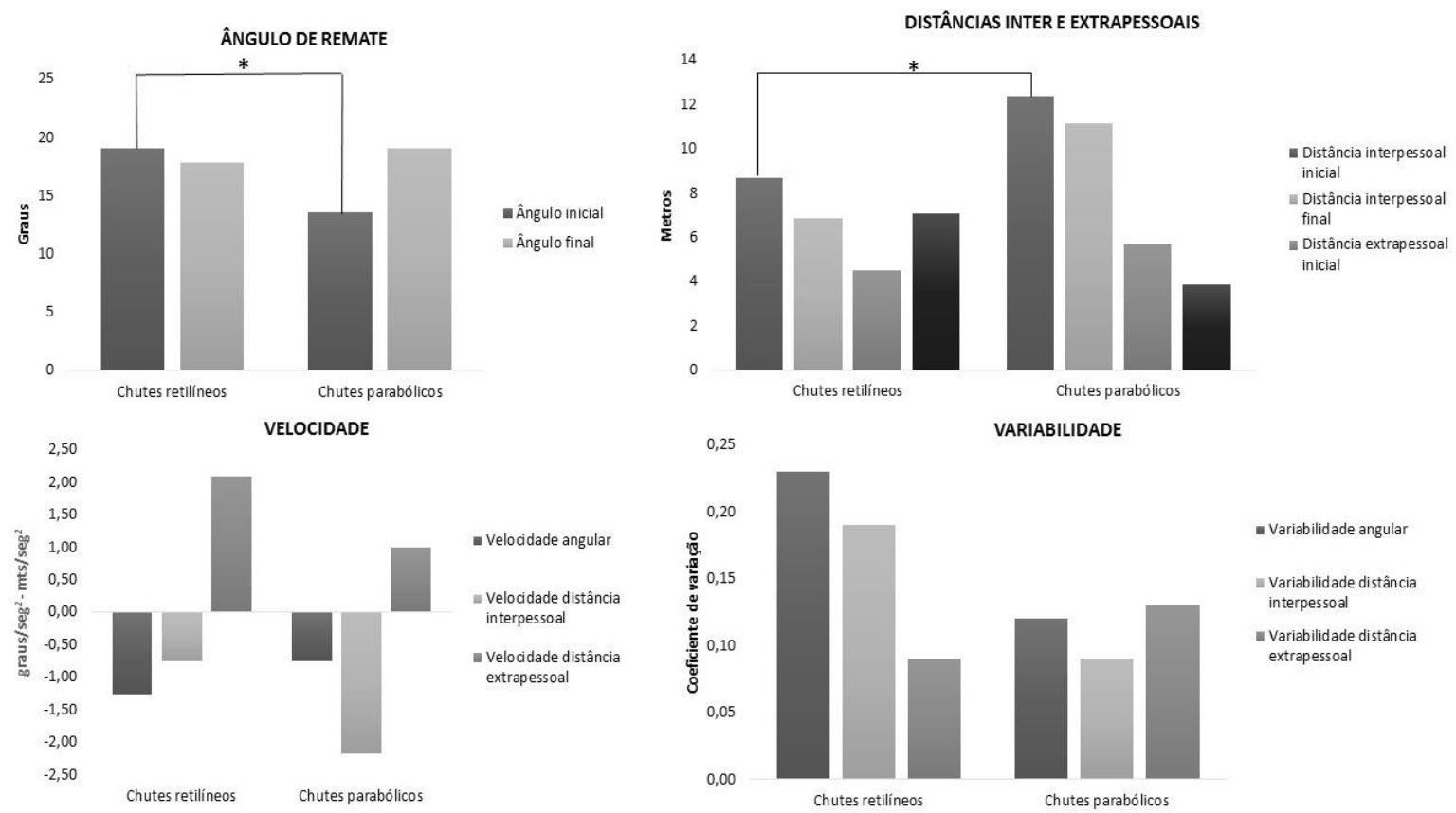

Figura 2. Médias dos ângulos de remate e distâncias inter e extrapessoais, velocidade e variabilidade em relação às trajetórias da bola.

\section{Segmento corporal}

Relativamente à parte do pé com que o remate foi realizado, os resultados mostraram diferenças significativas na variabilidade da $\mathrm{DE}$ entre as situações em que o remate foi realizado com a parte interna do pé e as situações em que o remate foi realizado com o peito do pé $\left(F_{(1,19)}=\right.$ 29.69, $p=0.00, \eta^{2}=0.60$ ). Conforme ilustra a Figura 3, os remates com a parte interna do pé forma realizados em condições de maior 
variabilidade da $\mathrm{DE}$ do que os remate realizados com a parte superior do pé. Nenhuma outra diferença estatisticamente significativa foi encontrada nas demais variáveis.

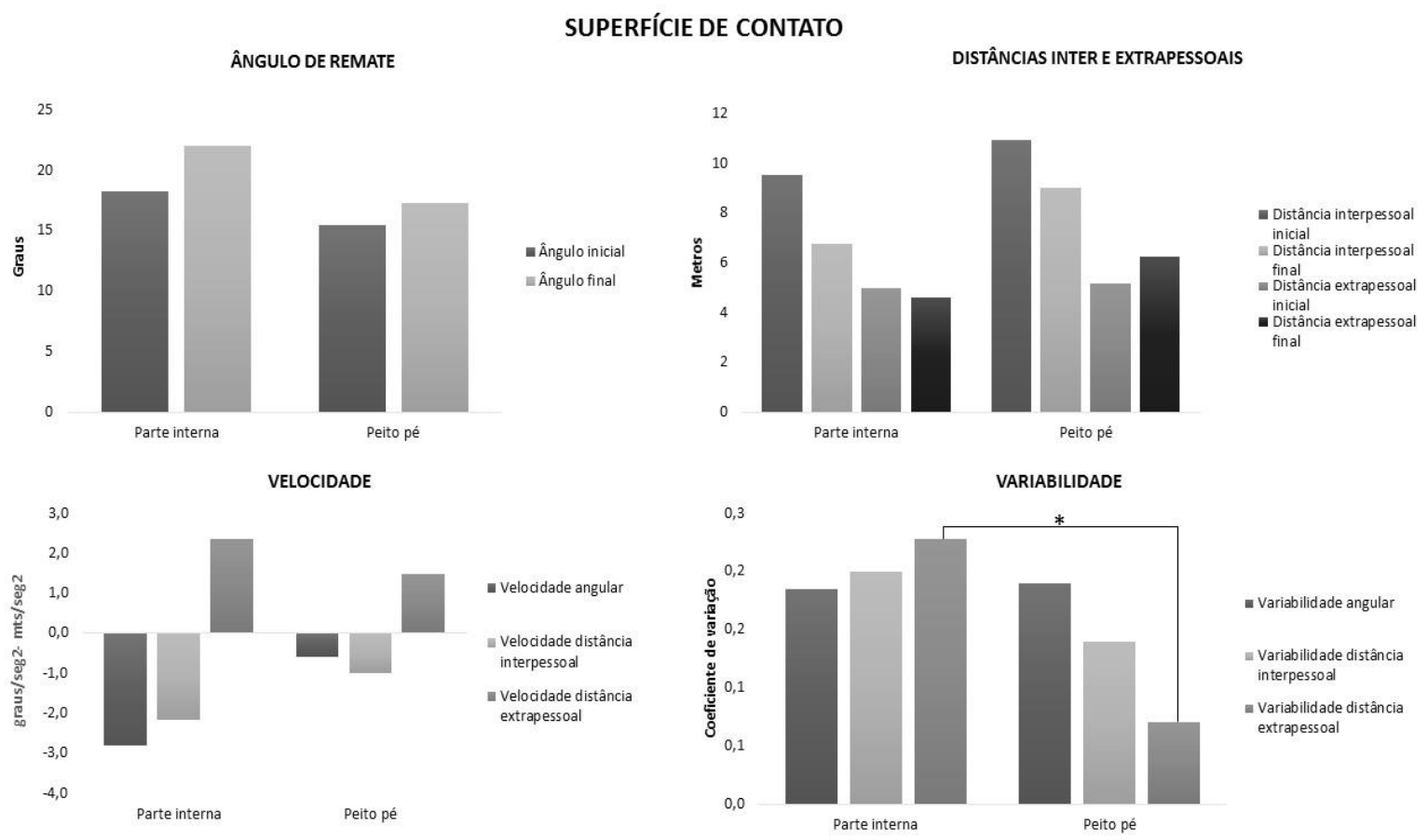

Figura 3. Médias dos ângulos de remate e distâncias inter e extrapessoais, velocidade e variabilidade em relação às superfícies de contato.

\section{DISCUSSÃO}

O presente estudo verificou as relações espaciais inter e extrapessoais como constrangimentos sobre a tomada de decisão relativa à trajetória da bola e à superfície de contato do pé com a bola na execução do remate dentro da grande área no futebol. Os resultados mostraram que os remates com trajetórias retilínea e parabólica foram executados com ângulos e distâncias interpessoais iniciais diferentes. E, os remates com as partes interna e superior do pé tiveram diferentes variabilidades de distância extrapessoal. Esses resultados permitem sugerir que a decisão sobre a trajetória da bola foi influenciada pelo ângulo inicial de remate, bem como pela distância inicial entre o jogador com posse de bola e o defensor mais próximo. Aliado a isto, os resultados também revelaram que a variabilidade da distância extrapessoal influenciou a escolha da superfície de contato. Especificamente, as situações em que a variabilidade da distância extrapessoal foi elevada, os jogadores decidiram chutar com a parte interna do pé, por outro lado, quando a variabilidade dessa medida foi menor os jogadores escolheram chutar com o peito do pé.

Os resultados mostraram que os jogadores escolheram uma trajetória retilínea nas situações em que a lacuna angular na baliza foi maior, no entanto, quando reduzida, a trajetória foi parabólica. Por ser o ângulo de remate uma medida pioneira deste estudo para a análise da trajetória da bola no futebol, não existem na literatura estudos comparativos com esta variável de análise. Ao levar em conta investigações em outros esportes que se utilizaram dessa medida, pode-se verificar evidências que vão ao encontro dos achados do presente estudo. Por exemplo, Corrêa et al. (2012, 2014) encontraram que a variável ângulo proporciona informações sobre as possiblidades de passe e de interceptação da bola, e atua como parâmetro de controle da direção e velocidade de execução do passe no futsal.

Além da lacuna angular na baliza no momento que o atacante recebeu a bola, a distância entre o atacante e o defensor mais próximo também se apresentou como constrangimento da trajetória da bola. Assim, no que diz respeito à distância interpessoal inicial, nas situações em que esta 
variável foi maior, os jogadores escolheram trajetórias parabólicas para o remate, o que encontra respaldo nos achados de Travassos et al. (2012) no futsal. Esses autores verificaram que nas situações em que o defensor estava mais afastado do atacante, ele tinha mais tempo para interceptar a bola e, portanto, maior possibilidade de sucesso. Desta forma, nossos achados permitem sugerir que quando a distância interpessoal inicial foi maior, os atacantes perceberam a possibilidade dos defensores em interceptar os remates com trajetórias retilíneas e escolheram trajetórias parabólicas, ou seja, as decisões da trajetória da bola estiveram influenciadas pelas possibilidades de sucesso da interceptação dos adversários.

Nossos resultados corroboram aqueles de Duarte et al. (2010) os quais também apontaram a variável distância como informação relevante em que os jogadores se baseiam para escolher a realização de ações no futebol. Especificamente, utilizando situações adaptadas 1 vs 1 em um espaço reduzido, esses autores mostraram que a distância entre o atacante e o defensor funcionou como constrangimentos do drible. No presente estudo, o qual foi realizado em um ambiente real de 11 vs 11, foi encontrado também que os valores angulares e a variabilidade da distância entre o defensor e a baliza influenciam na tomada de decisão dos jogadores.

Em relação à variabilidade da distância extrapessoal, quando esta foi menor, os jogadores escolheram o remate retilíneo ou parabólico com a parte superior do pé. Ao contrário disso, quando a variabilidade da distância extrapessoal foi maior, os jogadores decidiram chutar com a parte interna do pé independentemente da trajetória. Pensamos que o elevado nível na variabilidade desta distância aumenta a imprevisibilidade das situações, em razão disso, os jogadores utilizam a parte interna do pé para executar o remate, pois com esta superfície de contato, os remates são mais seguros e precisos. No entanto, mais evidências sobre estas variáveis são necessárias no futebol para fundamentar essa suposição. Vale lembrar que, nos estudos de Corrêa et al. (2012, 2014), foram encontrados resultados significativos sobre a variabilidade de uma medida angular na tomada de decisão no futsal, sendo esta outra evidência da importância do coeficiente de variação das medidas físicas como variável que restringe o comportamento dos jogadores nos esportes coletivos.

Dentre as limitações encontradas no presente estudo, pode se destacar a dificuldade para analisar alguns jogadores que participaram momentaneamente nas situações escolhidas, pois em alguns momentos do período em que foram realizados os rastreamentos, a localização destes esteve fora da área delimitada para a análise. Por outro lado, convém ressaltar que na literatura específica sobre a tomada de decisão da trajetória da bola e superfícies de contato no futebol, não existem até o presente momento, evidências sobre o uso das variáveis físicas ângulo de remate, distância entre o defensor e a baliza, assim como a velocidade e variabilidade. Desta forma, o presente trabalho caracteriza-se como pioneiro.

\section{CONCLUSÕES}

Os resultados do presente estudo nos permitem concluir que os jogadores utilizam as oportunidades de ação que as relações espaciais com os outros jogadores, a grande área e a baliza lhes oferecem, como informações relevantes para realizar suas escolhas sobre a trajetória e superfície de contato na execução do remate no futebol. Vale lembrar que, devido ao reduzido número de evidências, mais estudos nesta linha de investigação são necessários para o melhor entendimento da tomada de decisão, principalmente no futebol.

Nesse sentido, uma via de novas investigações pode estar direcionada à análise da relação entre as trajetórias da bola, superfícies de contato e a percentagem de sucesso de cada um deles. Aliado a isto, a utilização de outras medidas adicionais como a distância entre o atacante e a baliza, assim como a relação numérica entre jogadores de ataque e defesa, e a comparação das interações em situações de remate com outras ações realizadas dentro da grande área, podem trazer novas evidências para o melhor entendimento sobre as variáveis do ambiente do jogo que influenciam na tomada de decisão da execução do remate no futebol. 
Agradecimentos:

Nada a declarar

Conflito de Interesses:

Nada a declarar.

\section{Financiamento:}

Esta pesquisa foi financiada pela Fundação Capes

(Proex), Ministerio de educação do Brasil, concedida ao último autor.

\section{REFERÊNCIAS}

Araújo, D., Davids, K., \& Hristovski, R. (2006). The ecological dynamics of decision making in sport. Psychology of Sport and Exercise, 7(6), 653-676. doi: 10.1016/j.psychsport.2006.07.002

Bar-Eli, M., \& Raab, M. (2006). Judgment and decision making in sport and exercise: Rediscovery and new visions. Psychology of Sport and Exercise, $7(6)$, 519-524. doi: 10.1016/j.psychsport.2006.07.003

Corrêa, U. C., Davids, K., Silva, S. L., Denardi, R. A., \& Tani, G. (2014). The Influence of a Goalkeeper as an Outfield Player on Defensive Subsystems in Futsal. Advances in Physical Education, 4(2), 8492. doi: 10.4236/ape.2014.42012

Corrêa, U. C., Vilar, L., Davids, K., \& Renshaw, I. (2012). Informational constraints on the emergence of passing direction in the team sport of futsal. European Journal of Sport Science, 14(2), 1-8. doi: 10.1080/17461391.2012.730063

Corrêa, U. C., Vilar, L., Davids, K., \& Renshaw, I. (2014). Interpersonal Angular Relations between Players Constrain Decision-Making on the Passing Velocity in Futsal. Advances in Physical Education, 4(2), 93-101. doi: 10.4236/ape.2014.42013

Correia, V., Araújo, D., Duarte, R., Travassos, B., Passos, P., \& Davids, K. (2012). Changes in practice task constraints shape decision-making behaviours of team games players. Journal of Science and Medicine in Sport, 15(3), 244-249. doi: 10.1016/j.jsams.2011.10.004

Davids, K. (2009). The organization of action in complex neurobiological systems. In D. Araujo, H. Ripoll, \& M. Raab (Eds.), Perspectives on cognition and action in sport (3-13). New York: Nova Science, Inc.
Duarte, R., Freire, L., Gazimba, V., \& Araújo, D. (2010). A emergência da tomada de decisão no futebol: da decisão individual para a colectiva. In Nogueira, C. (Eds.), Psicologia Do Desporto: Actas do VII Simpósio Nacional de Investigação Em Psicologia. Braga: Universidade do Minho.

Esteves, P. T., de Oliveira, R. F., \& Araújo, D. (2011). Posture-related affordances guide attacks in basketball. Psychology of Sport and Exercise, 12(6), 639-644. doi: 10.1016/j.psychsport.2011.06.007

Fernandes, O., Folgado, H., Duarte, R., \& Malta, P. (2010). Validation of the tool for applied and contextual time-series observation. International Journal of Sport Psychology, 41, 63- 64.

Headrick, J., Davids, K., Renshaw, I., Araújo, D., Passos, P., \& Fernandes, O. (2011). Proximityto-goal as a constraint on patterns of behaviour in attacker-defender dyads in team games. Journal of Sports Sciences, 30(3), 247-253. doi: 10.1080/02640414.2011.640706

Millar, S.-K., Oldham, A. R., \& Renshaw, I. (2013). Interpersonal, intrapersonal, extrapersonal? Qualitatively investigating coordinative couplings between rowers in Olympic sculling. Nonlinear Dynamics, Psychology and Life Sciences, 17(3), 425-443.

Passos, P., Cordovil, R., Fernandes, O., \& Barreiros, J. (2012). Perceiving affordances in rugby union. Journal of Sports Sciences, 30(11), 1175-1182. doi: 10.1080/02640414.2012.695082

Travassos, B., Araújo, D., Davids, K., Vilar, L., Esteves, P., \& Vanda, C. (2012). Informational constraints shape emergent functional behaviours during performance of interceptive actions in team sports. Psychology of Sport and Exercise, 13(2), 216-223. doi: 10.1016/j.psychsport.2011.11.009

Travassos, B., Araújo, D., Duarte, R., \& McGarry, T. (2012). Spatiotemporal coordination behaviors in futsal (indoor football) are guided by informational game constraints. Human Movement Science, 31(4), 932-945. doi: 10.1016/j.humov.2011.10.004

Vilar, L., Araújo, D., Davids, K., \& Button, C. (2012). The role of ecological dynamics in analysing performance in team sports. Sports Medicine, 42(1), 1-10.

Vilar, L., Araújo, D., Davids, K., Correia, V., \& Esteves, P. T. (2013). Spatial-temporal constraints on decision-making during shooting performance in the team sport of futsal. Journal of Sports Sciences, 31(8), 840-846. doi: $10.1080 / 02640414.2012 .753155$ 